\title{
Demographic, Epidemiologic and Clinical Analyses of Paediatric Patients Hospitalized with Henoch- Schonlein Purpura: A Retrospective Study
}

\author{
(1) Şule Gökçe, (1) Burçe Emine Dörtkardeşler, (1) Aslı Aslan \\ Ege University Faculty of Medicine, Department of Pediatrics, General Pediatrics Unit, İzmir, Turkey
}

\begin{abstract}
Aim: Henoch-Schönlein purpura (HSP) is the most common systemic vasculitis in children. The purpose of this study was to assess the clinical, epidemiological, and laboratory features of 117 children diagnosed with HSP.

Materials and Methods: This study was conducted to evaluate the data of 117 children with HSP, retrospectively. The presence of the cardinal clinical findings such as purpura, abdominal pain, and arthralgia; and the presence of increased erythrocyte sedimentation rate, decreased serum C3 levels, leukocytosis, blood in the stool, haematuria, and proteinuria were assessed in children with HSP.

Results: Of the 117 patients enrolled in the study, 68 were males (58.1\%) and 49 were females (41.9\%). The median age was 85 (49) months. Purpuric skin lesions were detected in ninety-eight (97.9\%) patients, arthritis/arthralgia in 71 (60.6\%), gastrointestinal involvement in 52 (44.4\%), and renal involvement in 27 (23\%) patients. The median duration of hospitalization day was 6 (10) days. Non-palpable purpuric skin lesions mostly located on ankles were the most common involvement in the patients.

Conclusion: Non-palpable purpuric skin lesions were the most common presentations followed by arthritis/arthralgia, and gastrointestinal involvement in our patients. Seasonal distributions, gender, and ages of the patients were concluded to be noncontradictory compared with the literature. Although variable clinical findings in the patients were found, the cardinal features of HSP were determined in almost all subjects in this study.
\end{abstract}

Keywords: Henoch-Schöenlein purpura, children, vasculitis, systemic manifestation

\section{Introduction}

Henoch-Schönlein purpura (HSP), also known as immunoglobulin A (IgA) vasculitis which mediates a type III hypersensitivity reaction, is the most common vasculitis that affects the small vessels of the skin, joints, gastrointestinal tract, and kidneys in paediatric patients. HSP was primarily identified by Heberden in 1801 and described as having a relation with arthritis by Schonlein in 1837. The incidence of HSP is reported to be 10 cases per 100,000 yearly and it is generally seen during childhood between the ages of 5 to 15 years $(1,2)$.

This vasculitis is described by the triad of palpable purpura, abdominal pain, and arthritis. Purpuric skin lesions are the most common clinical findings in HSP followed by joint, gastrointestinal tract, and kidney involvement. 
Palpable cutaneous purpura is the major clinical diagnostic lesion mostly located on the lower extremities and buttocks. The second common involvement is arthritis which occurs in around $75 \%$ of patients. This arthritis generally affects the knees and ankles. Gastrointestinal involvement including colicky abdominal pain, vomiting, and gastrointestinal bleeding occurs in $50-75 \%$ of patients. Renal manifestation is usually seen as microscopic haematuria and nephriticnephrotic syndrome. Henoch-Schonlein purpura-associated involvements of neurological systems, cardiac systems, and the lung have also been reported during the disease in some studies (2).

Although the etiology of HSP cannot be clearly identified, the evidence of immunopathological mechanisms and possible trigger factors (upper respiratory system infections, drugs, vaccines, and other environmental exposures, etc.) have been reported in the literature (3). Leukocytoclastic vasculitis, which could be seen in other types of vasculitis, is characteristically demonstrated via skin biopsy. Renal and skin biopsies have displayed several abnormalities in IgA and IgA immune complexes $(3,4)$. HSP is usually a benign and self-limiting disease. Despite the fact that the disease is self-limiting clinically, due to the severity of some systemic involvements, and in rare cases mortality, physicians have sought to investigate it.

Various studies have focused on the function of regional and other environmental impacts in the etiology of HSP for many years. This study aimed to analyse the clinical features of those paediatric patients hospitalized with HSP, and evaluate the epidemiologic characteristics, demographic characteristics, and possible etiologic factors of HSP.

\section{Materials and Methods}

The medical records of 117 children with HSP were assessed retrospectively. The children were admitted to Ege University School of Medicine, Department of Paediatrics, General Paediatrics Unit, Izmir, Turkey between January 2013 and December 2019. The diagnosis of HSP was made in accordance with the Paediatric Rheumatology International Trials Organisation (PRINTO) 2005 criteria (5). This study was a continuation of a retrospective clinical study of HSP published in 2014 (DOI number 10.4274/jpr.40085). The Ethics Committee of the Ege University Hospital approved the study (20-1.1T/43).

Detailed demographic characteristics were obtained from the parents with a structured questionnaire. The medical records of these patients were evaluated by physicians and the clinical and laboratory data were extracted from patients' medical files. Studied variables including previous infections, vaccinations, and insect bites were recorded as precipitating factors.

As for the clinical characteristics, they were as follows: Microscopic haematuria was defined when the urine test result was $>5$ erythrocytes $/ \mathrm{mm}^{3}$; gross haematuria was defined when blood in the urine could be seen with the naked eye; rash location was determined as where purpura particularly accumulated on the parts of the body. Joint involvement was described as the presence of joint swelling and/or limitation of joint movement. Gastrointestinal involvement was identified as one of the following: abdominal pain, vomiting, hematemesis, melena, or positive stool test for occult blood. Recurrence was defined as a case of HSP which had been in remission for at least 1 month presenting with new lesions on the skin or with other systemic manifestations.

The laboratory data were analysed and the sedimentation rate was defined as elevated when it was $>20 \mathrm{~mm} /$ hour, and the presence of C3 levels $<900 \mathrm{mg} / \mathrm{L}$ were accepted as low. IgA level, C-reactive protein (CRP) level, anti-nuclear antibodies, albumin, and serum sodium levels were also obtained from all patients.

\section{Statistical Analysis}

Statistical analyses were performed using SPSS version 21.0 for personal computers (Chicago, IL, USA). Descriptive statistics were used to summarise the demographics and clinical features of the patients. Chi-square test and Fisher Exact test were used to assess associations between two or more qualitative variables. Continuous values were expressed as frequency (percentage) and mean \pm standard deviation or median and interquartile range (IQR); and when appropriate, categorical variables were determined by percentages.

\section{Results}

\section{Epidemiological Characteristics}

Table I shows the demographic and epidemiological data of the subjects. The study group included 68 males (58.1\%) and 49 females (41.9\%). Their ages ranged from 12 months to 16 years (median age 6 years). It was found that $42(35.8 \%)$ cases had been admitted to the ward during autumn (Figure 1). Prior to HSP onset, $50.6 \%$ of the patients had been recorded as being infected with an agent in their history, mostly upper respiratory tract infections. In regard to other trigger factors for HSP, 7 patients had enteritis, 3 had urinary tract infection, 1 patient was diagnosed with pneumonia. A predisposition factor was not found in $50.4 \%$ of the cases. 


\section{Clinical Characteristics}

The clinical characteristics of the 117 patients with HSP are shown in Table II. The typical rash with purpuric lesions concentrated mainly on the buttocks and the

Table I. Demographic and epidemiological data of 117 children with HSP

\begin{tabular}{|l|l|}
\hline Age, month, median (IQR) & $85(49)$ \\
\hline Gender & $68(58.1)$ \\
\hline Boys, n (\%) & $49(41.9)$ \\
\hline Girls, n (\%) &
\end{tabular}

Seasonal pattern, $\mathrm{n}(\%)$

\begin{tabular}{|l|l|}
\hline Autumn & $43(36.7)$ \\
\hline Winter & $32(27.3)$ \\
\hline Spring & $25(21.3)$ \\
\hline Summer & $17(14.5)$ \\
\hline
\end{tabular}

Etiologic factors, $\mathrm{n}(\%)$

\begin{tabular}{|l|l|}
\hline URTI & $46(39.3)$ \\
\hline Enteritis & $7(6)$ \\
\hline Pneumonia & $1(0.9)$ \\
\hline Urinary system infection & $3(2.6)$ \\
\hline Unknown & $59(50.4)$ \\
\hline $\begin{array}{l}\text { Duration of hospitalization, day, } \\
\text { median (IQR) }\end{array}$ & $\mathbf{6 ( 1 0 )}$ \\
\hline
\end{tabular}

City, n (\%)

\begin{tabular}{|l|l|}
\hline İzmir & $71(60.6)$ \\
\hline Manisa & $9(7.6)$ \\
\hline Balıkesir & $8(6.8)$ \\
\hline Muğla & $5(4.2)$ \\
\hline Uşak & $5(4.2)$ \\
\hline Aydın & $3(2.5)$ \\
\hline Other cities & $16(13.6)$ \\
\hline
\end{tabular}

URTI: Upper respiratory tract infection, SD: Standard deviation, IQR: Interquartile range, HSP: Henoch-Schönlein purpura

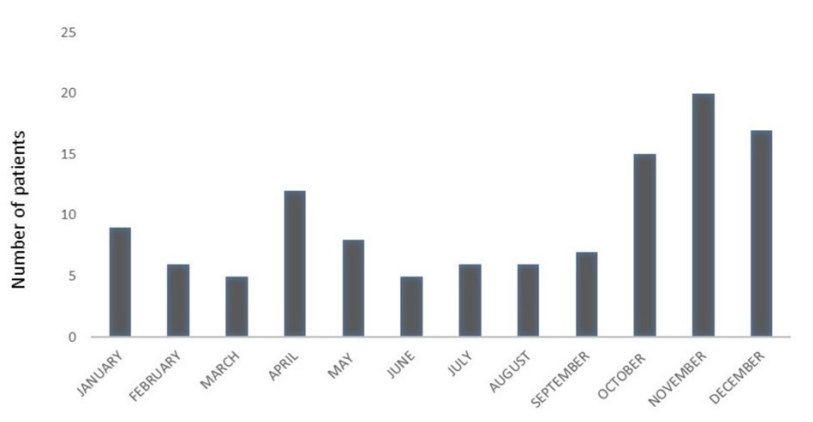

Figure 1. The seasonal pattern of patients lower extremities was observed in all the patients, except for 2 patients who had presented with gastrointestinal symptoms before the rash appeared. Almost all the patients (97.9\%) had the initial symptom, skin purpura, while it was accompanied by joint involvement in 71 (46.6\%) patients. The duration of the illness was 6 days as a median value in all the cases.

Table II. Main clinical features of the 117 children with HSP

Symptom

Cases (n) Percentage (\%)

First symptom

Skin purpura alone

Purpura plus joint pains

Purpura plus sole abdominal

pains

Purpura plus, abdominal pains and microscopic hematuria

Without purpura

25.6

\section{Rash location}

\begin{tabular}{|l|l|l|}
\hline Only LE & 37 & 31.6 \\
\hline LE plus buttocks & 44 & 37.6 \\
\hline LE plus buttocks plus UE & 12 & 10.3 \\
\hline LE plus UE & 8 & 6.8 \\
\hline LE plus buttocks plus trunk & 7 & 6 \\
\hline $\begin{array}{l}\text { LE plus buttocks plus trunk plus } \\
\text { face }\end{array}$ & 5 & 4.2 \\
\hline $\begin{array}{l}\text { LE plus buttocks plus trunk plus } \\
\text { face plus UE }\end{array}$ & 3 & 2.5 \\
\hline Without purpura & 1 & 0.8 \\
\hline Joint symptom (n=71) & &
\end{tabular}

Joint symptom $(n=71)$

Sole ankle involvement

Sole knee involvement

Knee plus ankle involvement

Multiple joint involvements

Digestive tract symptom $(n=52)$

\begin{tabular}{|l|l|}
\hline Abdominal pains & \\
\hline Vomiting & Occult blood in the stool \\
\hline Blood in the stool & \\
\hline
\end{tabular}

Kidney symptom $(n=27)$

\begin{tabular}{|l|l|l|}
\hline Microscopic hematuria & 15 & 55.5 \\
\hline $\begin{array}{l}\text { Macroscopic hematuria plus } \\
\text { proteinuria }\end{array}$ & 10 & 37.1 \\
\hline Gross hematuria & 2 & 7.4 \\
\hline LE: Lower extremities, UE: Upper extremities, HSP: Henoch-Schönlein purpura \\
\hline
\end{tabular}


A skin biopsy was obtained from 75 (64.1\%) patients. Thirty-five of the biopsy results showed classical leukocytoclastic vasculitis, and the others had non-specific features. Thirty-seven of 117 patients had localized edema usually appearing on the feet, scalp, hand, and scrotum. Joint involvement was the most common manifestation accompanying skin rashes. The joint regions are summarized in Table II. Twenty-seven (23\%) patients had renal involvement, these 27 cases can be further classified as follows: microscopic haematuria in 15 (55.5\%), gross haematuria in $2(7.4 \%)$, and haematuria plus proteinuria in 10 children (37\%). Renal biopsy was performed on 8 cases, one patient had focal mesangial proliferation with crescent lesions $<50 \%$, and the others had mesangial proliferation. Gastrointestinal involvement was detected in 52 (44.4\%) patients and the most common symptom was abdominal pain. The number of vomiting presentations of these patients was 30 (57.6\%) and 25 of the 52 patients with gastrointestinal involvement had positive occult blood in the stool. Two patients had been hospitalized with bloody stool. As an uncommon feature; orchitis was observed in 2 patients.

Those patients manifesting with arthritis were treated with $45 \mathrm{mg} / \mathrm{kg} /$ day ibuprofen and bed rest. Thirty-one of 117 HSP patients (93.3\%) were cured. Due to the fact that 32 patients had system involvements (renal or gastrointestinal), they were treated with low dose glucocorticoid medication. A pulse steroid treatment was started for two patients due to being unresponsive to low dose glucocorticoid treatment.

\section{Laboratory Findings (Table III)}

The mean serum IgA concentration was $217.8 \pm 93.7 \mathrm{mg} /$ $\mathrm{dL}$ and C-reactive protein levels ranged between $0-2 \mathrm{mg} / \mathrm{dL}$

\begin{tabular}{|l|l|}
\hline \multicolumn{2}{|l|}{ Table III. Laboratory findings of study group } \\
\hline Parameters & Results \\
\hline IgA (mean \pm SD) mg/dL & $217.8 \pm 93.7$ \\
\hline CRP [median (IQR)] mg/dL & $0.9(2.3)$ \\
\hline ESR mm/1 hour, n (\%) (>20) & $20(26.5)$ \\
\hline Albumin (mean \pm SD) mg/dL & $3.7 \pm 0.5$ \\
\hline C3 [median (IQR)] mg/dL & $134.5(36.5)$ \\
\hline C4 [median (IQR)] mg/dL & $25.5(14)$ \\
\hline Positive ANA, n (\%) & $15(12.8)$ \\
\hline Histopathology (n=75) & 35 (45.4) \\
\hline Leukocytoclastic vasculitis, n (\%) & $\begin{array}{l}\text { IgA: Immunoglobulin A, CRP: C-reactive protein, ESR: Erythrocyte } \\
\text { sedimentation rate, ANA: Anti-nuclear antibody, SD: Standard deviation, IQR: } \\
\text { Interquartile range }\end{array}$ \\
\hline
\end{tabular}

in $70(70.7 \%)$ patients. The erythrocyte sedimentation rate was found to be high in 20 children (26.5\%). Anti-nuclear antibody positivity was present in only $15(12.8 \%)$ patients without any other autoimmune disease features.

\section{Discussion}

HSP described as a triad: palpable purpura, abdominal pain, and arthritis, is the most common vasculitis in childhood. Although several pathogeneses of HSP have been reported, the exact etiopathogenesis is still a mystery. One of the most widely accepted pathogeneses is its association with the accumulation of "immune complexes containing IgA" and "complement components" in the walls of small vessels $(1,6)$. In this multisystemic disease, clinical features are usually self-limiting. However, the renal manifestations could result in chronic kidney disease in HSP patients (1).

In this study, we analysed the demographics, epidemiologic and clinical features of 117 children who were hospitalized with their first attack of HSP in Ege University Children's Hospital, General Paediatrics Unit. Similar to other epidemiologic studies for HSP, our study showed that the most common admission season was autumn. Several case series and longitudinal population-based surveys have reported that HSP in childhood is generally seen during both the autumn and winter seasons consistent with the literature $(7,8)$. Our reports showed that $50.6 \%$ of patients experienced infectious diseases, of which, the most common one was upper respiratory tract infection. Gastroenteritis and urinary system infections have also been proposed as a trigger factor for HSP. Several studies have reported other trigger events such as vaccinations, drugs, insect bites etc. $(1,9)$. Chen et al. (1) stated that $60.8 \%$ of their patients had an infection history before the onset of the clinical features of HSP as a possible predisposition factor. They also stated that food allergy or parasite infection may be a potential trigger factor for HSP (1). In our study, there were no relevant factors for HSP onset in 59 patients. Although there are a lot of suspected factors, the etiology of HSP has not been clearly determined yet.

HSP generally presents in children aged 5 to 15 years, predominantly in boys (10). In accordance with other studies, the demographic features of our study group showed that the median age was 85 months and more than half of the patients were male (58.1\%). Almost all of the patients with HSP were between the ages of 5-10 years. Anil et al. (11) reported a retrospective analysis of 430 HSP children in which they found that the mean age was 7.9 years The disease has generally been associated with a male dominance, however; some studies defined this feature as a reverse condition (12). 
Skin purpura alone was found as the first manifestation for HSP in $25.6 \%$ of the patients. All our patients showed the characteristic rashes on the lower extremities but some also on other trunk regions such as the arms or face. Four out of 117 patients had skin manifestations associated with bullous lesion concentrated on the lower extremities. The dominant initial symptom in this present study was skin rash and ankle involvement that required hospitalization for bed rest. Due to the fact that all our patients had not only skin manifestation but also joint involvement, our most frequent initial symptom was the coexistence of skin and joint involvement. The average time of the skin eruption was 14 days, ranging from 7 to 40 days in our patients. Chen et al. (1) determined that a majority of their HSP patients (69.1\%) displayed skin purpura alone as the first symptom, purpura with abdominal pain was described in $11.7 \%$ and purpura with joint pain in $9.2 \%$ of their patients. Dolezalova et al. (13) also determined palpable purpura as the main symptom at onset of HSP in their study population.

Gastrointestinal system (GIS) involvement may occur in $50 \%-80 \%$ of patients with mild-moderate symptoms such as vomiting, nausea, abdominal pain, and transient paralytic ileus (14). Gastrointestinal haemorrhage, bowel ischemia/necrosis, intussusception, bowel perforation have also been reported in HSP. Detection of severe gastrointestinal bleeding is an important evaluation as it might engender a life-threatening event in 15 to $35 \%$ of patients, especially precedence of the typical rash $(15,16)$. Before any skin manifestation is seen, bloody stool with abdominal pain may be initially treated as acute gastroenteritis (17). Intussusception is extremely rare, but it is the most common surgical indication for gastrointestinal involvement of HSP (18). Two cases in our study group had gastrointestinal bleeding which was not massive before skin manifestation. Cull et al. (19) investigated the clinical findings of 183 patients with HSP of whom 95 presented with GIS bleeding, and about $40 \%$ of their patients had bleeding before the typical lesions. Except for the bloody stool before purpura in two patients, our subjects did not show any rare presentations during the disease.

Renal involvement occurs in $40-50 \%$ of patients generally as microscopic haematuria and/or proteinuria. The manifestation of nephrotic syndrome or renal failure associated with renal involvement in HSP may manifest in a small percentage of patients. There is a risk of long-term renal sequelae in these patients and this risk increases with age. Renal histology generally shows a glomerular IgA deposition, endocapillary proliferation, epithelial crescents, subendothelial/subepithelial IgA deposits, and fibrin deposits. Trapani et al. (10) presented a study that evaluated 150 patients with HSP and 81 (54\%) children had had urinary abnormalities such as a mild nephropathy detected in 71 (47\%) cases: 42 patients with isolated mild proteinuria, 6 with microscopic isolated haematuria, and 23 patients with both abnormalities and normal renal function. Tabel et al. (6) reported that renal involvement was observed in $26.1 \%$ of their 107 HSP children. The authors have found that renal involvement was significantly more frequent in patients older than 10 years and that for the group with renal involvement, the frequency of scrotal involvement was significantly higher than for those in the group without renal involvement. Another study from western Turkey reported the renal involvement of HSP to be $44.7 \%$ in their cohort (11). During clinical follow-up in our study, our patients were examined for renal involvement by daily urinalysis and 15 (12.8\%) of 117 patients presented with microscopic haematuria and $2(1.7 \%)$ had macroscopic haematuria.

Although the dominant involvements of HSP are skin, joint, gastrointestinal, and kidney; infrequent and unusual system manifestations including cerebral vasculitis, scrotal or testicular haemorrhage, and interstitial pulmonary haemorrhage have been described. Neurological involvement of HSP might be present with obtundation, seizures, paresis, and posterior reversible encephalopathy syndrome, etc. There have also been reports of other interesting admission features of HSP in the literature such as orchitis, myositis, carditis, pulmonary haemorrhage, and anterior uveitis. Di Pietro et al. (20) reported a review of 23 paediatric cases with lung involvement associated with HSP. Those cases presenting with the pulmonary symptoms included cough, haemoptysis, epistaxis, dyspnea, tachypnea, chest pain, and shortness of breath through to acute respiratory failure. Therefore, atypical presentations affecting multiple systems should be considered in the outcome of HSP and the possibilities of different responses (20-22).

\section{Study Limitations}

There are some limitations to this study. The major limitation factor was the small number of patients. The first reason is that we did not include those patients treated and followed up in the outpatient policlinics and emergency services of our centre. The second limitation is that as a general rule in our outpatient policlinics, patients with HSP associated with skin manifestation alone are not hospitalized. Also, we did not focus on the identification of infectious agents that could have been associated with the triggering of HSP. It could have given clinicians an idea of 
which agent might be the trigger factor. Finally, we could not obtain skin biopsy from all study groups which could have ruled out other vasculitic disorders.

\section{Conclusion}

HSP is the most common vasculitis and it may be associated with risks of various complications in childhood. The underlying mechanisms of HSP's characteristic findings have not been exactly defined yet. This index study presents a descriptive analyse of paediatric patients in Ege University Children's hospital during a 6-year-period. Further studies are needed to investigate the influence of its pathogenesis in these cases and to find new mechanism that may play a role in following up children with HSP.

\section{Ethics}

Ethics Committee Approval: The Ethics Committee of the Ege University Hospital approved the study (20-1.1T/43).

Informed Consent: Informed consents were not required because the study was conducted retrospectively.

Peer-review: Externally peer-reviewed.

\section{Authorship Contributions}

Data Collection or Processing: S.G., B.E.D., A.A., Analysis or Interpretation: Ş.G., B.E.D., A.A.,Writing: Ş.G., B.E.D.

Conflict of Interest: No conflict of interest was declared by the authors.

Financial Disclosure: The authors have no financial relationships relevant to this article to disclose.

\section{References}

1. Chen $O$, Zhu XB, Ren P, Wang YB, Sun RP, Wei DE. Henoch Schonlein Purpura in children: clinical analysis of 120 cases. Afr. Health Sci 2013; 13:94-9.

2. Saulsbury FT. Clinical update: Henoch-Schönlein purpura. Lancet 2007; 369:976-8.

3. Yang $\mathrm{YH}$, Chuang $\mathrm{YH}$, Wang LC, Huang HY, Gershwin ME, Chiang BL. The immunobiology of Henoch-Schonlein purpura. Autoimmun Rev 2008; 7:179-84.

4. Ozaltin F, Bakkaloglu A, Ozen S, et al. The significance of IgA class antineutrophil cytoplamsic antibodies (ANCA) in childhood Henoch-Schonlein purpura. Clin Rheumatol 2004; 23:426-29.

5. Ozen S, Pistorio A, lusan SM, et al. EULAR/PRINTO/PRES criteria for Henoch-Schönlein purpura, childhood polyarteritis nodosa, childhood Wegener granulomatosis and childhood Takayasu arteritis: Ankara 2008. Part II: Final classification criteria. An Rheum Dis 2010; 69:798-806.
6. Tabel $Y$, Inanc FC, Dogan DG, Elmas AT. Clinical features of children with Henoch-Schonlein purpura: risk factors associated with renal involvement. Iran J Kidney Dis 2012; 6:269-74.

7. Piram M, Mahr A. Epidemiology of immunoglobulin A vasculitis (Henoch-Schonlein): current state of knowledge. Curr Opin Rheumatol 2013; 25:171-78.

8. Penny K, Fleming M, Kazmierczak D, Thomas A. An epidemiological study of Henoch-Schonlein purpura. Paediatr Nurs 2010; 22:30-5.

9. Martinez Lopez MM, Rodriguez Arranz C, Pena Carrion A, Merino Munoz R, Garcia-Consuegra Molina J. Henoch-Schonlein purpura. Study of factors associated with the development and course of the disease. An Pediatr (Barc) 2007; 66:453-8.

10. Trapani S, Micheli A, Grisolia F, et al. Henoch Schonlein purpura in childhood: epidemiological and clinical analysis of 150 cases over a 5 -year period and review of literature. Semin Aarthritis Rheum 2005; 35:143-53.

11. Anil M, Aksu N, Kara OD, et al. Henoch- Schonlein purpura in children from western Turkey: a retrospective analysis of 430 cases. Turk J Pediatr 2009; 51:429-36.

12. Garcia-Porrua C, Calvino MC, Llorca I, Couselo JM, GonzalezGay MA. Henoch-Schonlein purpura in children and adults: clinical differences in a defined population. Semin Aarthritis Rheum 2002; 32:149-56.

13. Dolezalova $P$, Telekesova $P$, Nemcova D, Hoza ). Incidence of vasculitis in children in the Czech Republic: 2-year prospective epidemiology survey. I Rheumatol 2004; 31:2295-9.

14. Chang $\mathrm{WL}$, Yang $\mathrm{YH}$, Lin $\mathrm{YT}$, Chiang BL. Gastrointestinal manifestations in Henoch-Schönlein purpura: a review of 261 patients. Acta Paediatr 2004; 93:1427-31.

15. Wu CS, Tung SY. Henoch-Schönlein purpura complicated by upper gastrointestinal bleeding with an unusual endoscopic picture. J Clin Gastroenterol 1994; 19:128-31.

16. Gunasekaran TS, Berman J, Gonzalez M. Duodenojejunitis: is it idiopathic or is it Henoch-Schönlein purpura without the purpura? I Pediatr Gastroenterol Nutr 2000; 30:22-8.

17. Lin S), Chao HC, Huang IL. Gastrointestinal involvement as the initial manifestation in children with Henoch-Schönlein purpura-clinical analysis of 27 cases. Zhonghua Min Guo Xiao Er Ke Yi Xue Hui Za Zhi 1998; 39:186-90.

18. Lai HC. Henoch-Schonlein purpura with intussusception: a case report. Pediatr Neonatol 2010; 51:65-7.

19. Cull DL, Rosario V, Lally KP, Ratner I, Mahour GH. Surgical implications of Henoch-Schönlein purpura. Pediatr Surg 1990; 25:741-3.

20. Di Pietro GM, Castellazzi ML, Mastrangelo A, Montini G, Marchisio P, Tagliabue C. Henoch-Schönlein Purpura in children: not only kidney but also lung. Pediatr Rheumatol Online I 2019; 17:75.

21. Lava SAG, Peeters GG, Bianchetti MG, Simonetti BG, Simonetti $G D$, Milani GP. Posterior reversible encephalopathy syndrome in Henoch-Schönlein purpura, Rheumatol Int 2017; 37:461-3.

22. Andrew Brodie, Natasha G, Nitiahpapand R, Chowoo L. Unusual presentation of Henöch-Schonlein purpura. BMJ Case Rep 2018; 30:bcr2017220129. 